Article

\title{
Domain of Existence and Uniqueness for Nonlinear Hammerstein Integral Equations ${ }^{\dagger}$
}

\author{
Sukhjit Singh ${ }^{1}$, Eulalia Martínez ${ }^{2, *}$, Abhimanyu Kumar ${ }^{3}$ and D. K. Gupta ${ }^{4}$ \\ 1 National Institute of Technology, Jalandhar 144011, India; sukhjitmath@gmail.com \\ 2 Instituto de Matemática Multidisciplinar, Universitat Politècnica de València, 46022 Valencia, Spain \\ 3 Department of Mathematics, L. N. M. U. Darbhanga-Bihar, Darbhanga 846004, India; \\ abhimanyu.kumar01991@iitkgp.ac.in \\ 4 Department of Mathematics, I.I.T Kharagpur, Kharagpur 721302, India; dkg@maths.iitkgp.ernet.in \\ * Correspondence: eumarti@mat.upv.es \\ + This paper is an extended version of our paper published in Mathematical Modelling in Engineering and \\ Human Behavior 2017.
}

Received: 14 February 2020; Accepted: 3 March 2020; Published: 9 March 2020

check for updates

\begin{abstract}
In this work, we performed an study about the domain of existence and uniqueness for an efficient fifth order iterative method for solving nonlinear problems treated in their infinite dimensional form. The hypotheses for the operator and starting guess are weaker than in the previous studies. We assume omega continuity condition on second order Fréchet derivative. This fact it is motivated by showing different problems where the nonlinear operators that define the equation do not verify Lipschitz and Hölder condition; however, these operators verify the omega condition established. Then, the semilocal convergence balls are obtained and the R-order of convergence and error bounds can be obtained by following thee main theorem. Finally, we perform a numerical experience by solving a nonlinear Hammerstein integral equations in order to show the applicability of the theoretical results by obtaining the existence and uniqueness balls.
\end{abstract}

Keywords: semilocal convergence; Lipschitz condition; Hölder condition; Hammerstein integral equation; dynamical systems

MSC: 65G49; 47H99

\section{Introduction}

Let $X$ and $Y$ are Banach spaces where $G: \Omega \subseteq X \rightarrow Y$ be a nonlinear function in an open convex domain $\Omega_{0} \subseteq \Omega$. We use iterative methods in order to solve the nonlinear equation:

$$
G(x)=0
$$

which characterizes various real life problems such as dynamical systems, boundary value problems described by ordinary differential equations, partial derivative equations and nonlinear integral equations with applications in different fields of engineering, finances, optimization costs and benefits, etc. A great variety of iterative schemes for solving these problems are obtained in [1-3]. It is well known that Newton's method is the most widely used iterative scheme to solve (1), it is defined for $k \geq 0$, by

$$
x_{k+1}=x_{k}-\Gamma_{k} G\left(x_{k}\right)
$$


where $\Gamma_{k}=G^{\prime}\left(x_{k}\right)^{-1}$ and $x_{0}$ is the starting guess and it reaches convergence order two. Different modifications of Newton's method have been published in order to increase the order of convergence and efficiency. We center in such publications in the literature involving complete studies in the sense of local and semilocal convergence, (see, [4-19]), where authors studied the convergence of iterative methods with high order of convergence under different continuity hypotheses.

In a recent paper, Singh et al. [20] presented the semilocal convergence of an efficient fifth order method under the Lipschitz condition on second derivative for non linear operator $F^{\prime \prime}$. The iterative scheme can be written for $k=0,1,2 \ldots$ as

$$
\begin{aligned}
y_{k} & =x_{k}-\Gamma_{k} G\left(x_{k}\right), \\
z_{k} & =y_{k}-\Gamma_{k} G\left(y_{k}\right), \\
x_{k+1} & =z_{k}-G^{\prime}\left(y_{k}\right)^{-1} G\left(z_{k}\right) .
\end{aligned}
$$

In real life applications, (see [21-23]), various numerical examples involving Hammerstein type integral equation [2] can be found which neither satisfy the Lipschitz nor the Hölder condition. This is the reason that motivated us to establish the semilocal convergence for the iterative method defined above in (3) under weaker conditions, which is also an efficient fifth-order method.

Consider nonlinear Hammerstein type integral equation

$$
x(r)+\sum_{i=1}^{m} \int_{a}^{b} K_{i}(r, s) S_{i}(x(s)) d s=f(r), r \in[a, b],
$$

where functions $f, K_{i}$ and $S_{i}$ for $i=1,2, \ldots m$ are known, the solution $x$ is to be determined and $-\infty<a<b<+\infty$. In order to solve (4), we have to solve

$$
G(x)(u)=x(u)+\sum_{i=1}^{m} \int_{a}^{b} K_{i}(u, v) S_{i}(x(v)) d v-f(u)
$$

If $S_{i}^{\prime}(x(u))$ is $\left(M_{i}, \alpha_{i}\right)$ - Hölder continuous in $\Omega$, then, under max-norm, we have

$$
\left\|G^{\prime \prime}(x)-G^{\prime \prime}(y)\right\| \leq \sum_{i=1}^{m} M_{i}\|x-y\|^{\alpha_{i}}, M_{i} \geq 0, \alpha_{i} \in[0,1], \forall x, y \in \Omega .
$$

For different $\alpha_{i}, G^{\prime \prime}$ neither satisfies Lipschitz nor Hölder condition but satisfies the weaker $\omega$-condition.

In this work and in Section 2, we developed the semilocal convergence analysis of an iterative method of five order of convergence; this has been done under weaker conditions for solving nonlinear equations. Moreover, theoretical results about the existence and uniqueness for the solution have been established along with error bounds for the solution. In Section 3, we developed numerical examples and obtained the radius of existence and uniqueness for the solution, showing the applicability of our study. Finally, some conclusions are included in Section 4.

\section{Semilocal Convergence Analysis}

In this section, we give the hypothesis for the nonlinear operator $\mathrm{G}$ in the starting point, so we construct the convergence ball centered at this point, that is, the ball at which all the iterates belong and converge to the solution.

\subsection{Preliminaries Results}

Let $x_{0} \in \Omega$, such as $\Gamma_{0}=G^{\prime}\left(x_{0}\right)^{-1} \in B L(Y, X)$ exists, being a bounded linear operator from $Y$ to $X$ for which the following conditions hold. 
(1) $\left\|\Gamma_{0}\right\| \leq \beta_{0}$

(2) $\left\|\Gamma_{0} G\left(x_{0}\right)\right\| \leq \eta_{0}$

(3) $\left\|G^{\prime \prime}(x)\right\| \leq M$

(4) $\left\|G^{\prime \prime}(x)-G^{\prime \prime}(y)\right\| \leq \omega(\|x-y\|), x, y \in \Omega$, for a non-decreasing continuous real function $\omega(a)$, $a>0, \omega(0) \geq 0$ such that, $\omega(t a) \leq t^{q} \omega(a)$ for $t \in[0,1], a \in(0, \infty)$ and $q \in[0,1]$.

Let $r_{0}=M \beta_{0} \eta_{0}, s_{0}=\beta_{0} \eta_{0} \omega\left(\eta_{0}\right)$ and define sequences $\left\{r_{k}\right\},\left\{s_{k}\right\}$ and $\left\{\eta_{k}\right\}$ for $k=0,1,2 \ldots$, by

$$
\begin{aligned}
r_{k+1} & =r_{k} \phi\left(r_{k}\right)^{2} \psi\left(r_{k}, s_{k}\right), \\
s_{k+1} & =s_{k} \phi\left(r_{k}\right)^{2+q} \psi\left(r_{k}, s_{k}\right)^{1+q}, \\
\eta_{k+1} & =\eta_{k} \phi\left(r_{k}\right) \psi\left(r_{k}, s_{k}\right),
\end{aligned}
$$

where,

$$
\begin{aligned}
\phi(u) & =\frac{1}{1-u g(u)} \\
g(u) & =\left(1+\frac{u}{2}+\frac{u^{2}}{2(1-u)}\left(1+\frac{u}{4}\right)\right)
\end{aligned}
$$

and

$$
\begin{aligned}
\psi(u, v) & =\frac{u^{2}}{2(1-u)}\left(1+\frac{u}{4}\right)\left[\frac{v}{1+q}\left(\frac{u^{1+q}}{2^{1+q}}+\frac{1}{2+q}\left(\frac{u^{2}}{2(1-u)}\left(1+\frac{u}{4}\right)\right)^{1+q}\right)\right. \\
& \left.+\frac{u}{2}\left(u+\frac{u^{2}}{2(1-u)}\left(1+\frac{u}{4}\right)\right)\right] .
\end{aligned}
$$

Let $h(u)=g(u) u-1$. Moreover, $h(0)=-1$ and $g(u)$ is an increasing function, therefore, $h(u)$ has a real root $v$ verifying If $u \in(0, v)$, we get $g(u) u<1$.

Lemma 1. Let $\phi(u), g(u)$ and $\psi(u, v)$ are given by (10), (11) and (13) respectively. If $0<r_{0}<v$ and $\phi\left(r_{0}\right)^{2} \psi\left(r_{0}, s_{0}\right)<1$, then

(i) $\phi(u)$ and $g(u)$ are increasing functions verifying $\phi(u)>1, g(u)>1$ for $u \in(0, v)$.

(ii) $\psi(u, v)$ is an increasing function of $u$, for $u \in(0, v)$.

(iii) $\left\{r_{k}\right\},\left\{s_{k}\right\}$ and $\left\{\eta_{k}\right\}$ are decreasing sequences and $r_{k} g\left(r_{k}\right)<1$ as well as $\phi\left(r_{k}\right)^{2} \psi\left(r_{k}, s_{k}\right)<1$ for $k \geq 0$.

Proof. The proof of $(i)$ and (ii) are trivial. The proof of (iii) can be given in the following manner. For $k=0$, (7) gives $r_{1}=r_{0} \phi\left(r_{0}\right)^{2} \psi\left(r_{0}, s_{0}\right)<r_{0}$. Using (8) and (9), we get $s_{1}=s_{0} \phi\left(r_{0}\right)^{2+q} \psi\left(r_{0}, s_{0}\right)^{1+q}<$ $s_{0}\left(\phi\left(r_{0}\right)^{2} \psi\left(r_{0}, s_{0}\right)\right)^{1+q}<s_{0}$ and $\eta_{1}=\phi\left(r_{0}\right) \psi\left(r_{0}, s_{0}\right) \eta_{0}<\eta_{0}$. Thus, (iii) holds for $k=0$. Since, $\phi(u)$ and $g(u)$ are increasing functions, and therefore, by using mathematical induction Lemma 1 holds $\forall k \geq 0$.

Lemma 2. Let $\phi(u)$ and $\psi(u, v)$ be defined by (10) and (13). If $\gamma \in(0,1)$ we have $\phi(\gamma t)<\gamma \phi(t)$ and $\psi\left(\gamma u, \gamma^{1+q} v\right)<\gamma^{3+q} \psi(u, v)$.

Proof. The proof is trivial. Since $g(\gamma t)<g(t)$, as $g(t)$ is an increasing function. Therefore, $\phi(\gamma t)<$ $\phi(t)$. Now, 


$$
\begin{aligned}
\psi\left(\gamma u, \gamma^{1+q} v\right) & =\frac{(\gamma u)^{2}}{2(1-\gamma u)}\left(1+\frac{\gamma u}{4}\right)\left[\frac{\left(\gamma^{1+q} v\right)}{1+q}\left(\frac{(\gamma u)^{1+q}}{2^{1+q}}+\frac{1}{2+q}\left(\frac{(\gamma u)^{2}}{2(1-\gamma u)}\left(1+\frac{\gamma u}{4}\right)\right)^{1+q}\right)\right. \\
& \left.+\frac{(\gamma u)}{2}\left(\gamma u+\frac{(\gamma u)^{2}}{2(1-\gamma u)}\left(1+\frac{\gamma u}{4}\right)\right)\right] . \\
& <\gamma^{3+q} \psi(u, v)
\end{aligned}
$$

Lemma 3. Let $\gamma=\phi\left(r_{0}\right)^{2} \psi\left(r_{0}, s_{0}\right), 0<r_{0}<v$ and $\delta=\frac{1}{\phi\left(r_{0}\right)}$. Then,

(i) $r_{k} \leq \gamma^{(4+q)^{k-1}} r_{k-1} \leq \gamma^{\frac{(4+q)^{k}-1}{3+q}} r_{0}$ and $s_{k} \leq\left(\gamma^{(4+q)^{k-1}}\right)^{1+q} s_{k-1} \leq\left(\gamma^{\frac{(4+q)^{k}-1}{3+q}}\right)^{1+q} s_{0}$.

(ii) $\phi\left(r_{k}\right) \psi\left(r_{k}, s_{k}\right) \leq \frac{\gamma^{(4+q)^{k}}}{\phi\left(r_{0}\right)} \forall k \in N$.

(iii) $\eta_{k} \leq \gamma^{\frac{(4+q)^{k}-1}{3+q}} \delta^{k} \eta_{0}$.

Proof. Using $k=0$ in (7) and (8), we get $r_{1}=r_{0} \phi\left(r_{0}\right)^{2} \psi\left(r_{0}, s_{0}\right)=\gamma r_{0}$ and

$$
s_{1}=s_{0} \phi\left(r_{0}\right)^{2+q} \psi\left(r_{0}, s_{0}\right)^{1+q} \leq \gamma^{1+q} s_{0} .
$$

Thus, Lemma holds for $k=0$. Assume that Lemma holds for $k=n$. Using induction, we will prove for $k=n+1$. Then, we have

$$
\begin{aligned}
r_{n+1} & =r_{n} \phi\left(r_{n}\right)^{2} \psi\left(r_{n}, s_{n}\right) \\
& \leq \gamma^{(4+q)^{n-1}} r_{n-1} \phi\left(\gamma^{(4+q)^{n-1}} r_{n-1}\right)^{2} \psi\left(\gamma^{(4+q)^{n-1}} r_{n-1}\left(\gamma^{(4+q)^{n-1}}\right)^{1+q} s_{n-1}\right) \\
& \leq \gamma^{(4+q)^{n-1}} r_{n-1} \phi\left(r_{n-1}\right)^{2}\left(\gamma^{(4+q)^{n-1}}\right)^{3+q} \psi\left(r_{n-1}, s_{n-1}\right) \\
& \leq\left(\gamma^{(4+q)^{n-1}}\right)^{(4+q)} r_{n-1} \phi\left(r_{n-1}\right)^{2} \psi\left(r_{n-1}, s_{n-1}\right), \\
& \leq \gamma^{(4+q)^{n}} r_{n} .
\end{aligned}
$$

In a similar manner, we get

$$
\begin{aligned}
r_{n+1} & \leq \gamma^{(4+q)^{n}} r_{n} \leq \gamma^{(4+q)^{n}} \gamma^{(4+q)^{n-1}} r_{n-1} \\
& \leq \cdots \leq \gamma^{(4+q)^{n}} \gamma^{(4+q)^{n-1}} \ldots \gamma^{(4+q)^{0}} r_{0}=\gamma^{\frac{(4+q)^{n+1}-1}{3+q}} r_{0} .
\end{aligned}
$$

Now, we consider

$$
\begin{aligned}
s_{n+1} & =s_{n} \phi\left(r_{n}\right)^{(2+q)} \psi\left(r_{n}, s_{n}\right)^{1+q} \leq s_{n}\left(\phi\left(r_{n}\right)^{2} \psi\left(r_{n}, s_{n}\right)\right)^{1+q} \\
& \leq s_{n}\left(\frac{r_{n+1}}{r_{n}}\right)^{1+q} \leq\left(\gamma^{(4+q)^{n}}\right)^{1+q} s_{n}
\end{aligned}
$$


proceeding in this way, we get

$$
\begin{aligned}
s_{n+1} & \leq\left(\gamma^{(4+q)^{n}}\right)^{1+q} s_{n} \leq\left(\gamma^{(4+q)^{n}}\right)^{1+q}\left(\gamma^{(4+q)^{n-1}}\right)^{1+q} s_{n-1} \\
& \leq\left(\gamma^{\frac{(4+q)^{n+1}-1}{3+q}}\right)^{1+q} s_{0} .
\end{aligned}
$$

Hence, ( $i$ ) holds $\forall k \geq 0$ by using mathematical induction. Now, consider

$$
\begin{aligned}
\phi\left(r_{k}\right) \psi\left(r_{k}, s_{k}\right) & \leq \phi\left(\gamma^{\frac{(4+q)^{k}-1}{3+q}} r_{0}\right) \psi\left(\gamma^{\frac{(4+q)^{k}-1}{3+q}} r_{0},\left(\gamma^{\frac{(4+q)^{k}-1}{3+q}}\right)^{1+q} s_{0}\right) \\
& \leq \gamma^{(4+q)^{k}-1} \phi\left(r_{0}\right) \psi\left(r_{0}, s_{0}\right)=\gamma^{(4+q)^{k}} \delta .
\end{aligned}
$$

Thus, (ii) is proven. From (9), we get

$$
\begin{aligned}
\eta_{k} & =\phi\left(r_{k-1}\right) \psi\left(r_{k-1}, s_{k-1}\right) \eta_{k-1} \leq \prod_{n=0}^{k-1} \phi\left(r_{n}\right) \psi\left(r_{n}, s_{n}\right) \eta_{0} \\
& \leq \prod_{n=0}^{k-1} \frac{\gamma^{(4+q)^{n}}}{\phi\left(r_{0}\right)} \eta_{0} \leq \gamma^{\frac{(4+q)^{k}-1}{1+q}} \delta^{k} \eta_{0} .
\end{aligned}
$$

Thus, (iii) is proven.

\subsection{Main Results}

In this section, we establish the recurrence relations for (3) under the assumption considered in the previous section. Consider

$$
\left\|I-\Gamma_{0} G^{\prime}\left(y_{0}\right)\right\| \leq\left\|\Gamma_{0}\right\|\left\|G^{\prime}\left(y_{0}\right)-G^{\prime}\left(x_{0}\right)\right\| \leq M \beta_{0} \eta_{0}=r_{0},
$$

if $r_{0}<1$, then

$$
\left\|G^{\prime}\left(y_{0}\right)^{-1} G^{\prime}\left(x_{0}\right)\right\| \leq \frac{1}{1-r_{0}}
$$

and by substituting $k=0$ in (3), we get

$$
z_{0}-x_{0}=-\Gamma_{0} G\left(x_{0}\right)-\Gamma_{0} G\left(y_{0}\right) .
$$

By using Taylor expansion of $G\left(y_{0}\right)$ about $x_{0}$, we get

$$
\begin{aligned}
G\left(y_{0}\right) & =G\left(x_{0}\right)+G^{\prime}\left(x_{0}\right)\left(y_{0}-x_{0}\right)+\int_{0}^{1} G^{\prime \prime}\left(x_{0}+\theta\left(y_{0}-x_{0}\right)\right)\left(y_{0}-x_{0}\right)^{2}(1-\theta) d \theta \\
& =\int_{0}^{1} G^{\prime \prime}\left(x_{0}+\theta\left(y_{0}-x_{0}\right)\right)\left(y_{0}-x_{0}\right)^{2} \theta d \theta
\end{aligned}
$$

Then, by using (21) in (20) and taking norm, we get

$$
\begin{aligned}
\left\|z_{0}-x_{0}\right\| & \leq\left\|\Gamma_{0} G\left(x_{0}\right)\right\|+\|\Gamma\| \frac{M}{2}\left\|y_{0}-x_{0}\right\|^{2} \\
& \leq\left(1+\frac{r_{0}}{2}\right) \eta_{0} .
\end{aligned}
$$

Now,

$$
\left\|z_{0}-y_{0}\right\|=\left\|\Gamma_{0} G\left(y_{0}\right)\right\| \leq \frac{r_{0}}{2} \eta_{0}
$$


and by substituting $k=0$ in (3) and taking norm, we get

$$
\begin{aligned}
\left\|x_{1}-z_{0}\right\| & \leq\left\|G^{\prime}\left(y_{0}\right)^{-1} G\left(z_{0}\right)\right\| \leq\left\|G^{\prime}\left(y_{0}\right)^{-1} G^{\prime}\left(x_{0}\right)\right\|\left\|\Gamma_{0} G\left(z_{0}\right)\right\| \\
& \leq \frac{r_{0}^{2}}{2\left(1-r_{0}\right)}\left(1+\frac{r_{0}}{4}\right) \eta_{0} .
\end{aligned}
$$

Therefore,

$$
\begin{aligned}
\left\|x_{1}-x_{0}\right\| & \leq\left\|x_{1}-z_{0}\right\|+\left\|z_{0}-x_{0}\right\| \\
& \leq\left(1+\frac{r_{0}}{2}+\frac{r_{0}^{2}}{2\left(1-r_{0}\right)}\left(1+\frac{r_{0}}{4}\right)\right) \eta_{0}=g\left(r_{0}\right) \eta_{0} .
\end{aligned}
$$

So, we have

$$
\begin{aligned}
\left\|I-\Gamma_{0} G^{\prime}\left(x_{1}\right)\right\| & \leq\left\|\Gamma_{0}\right\|\left\|G^{\prime}\left(x_{1}\right)-G^{\prime}\left(x_{0}\right)\right\| \leq \beta_{0} M\left\|x_{1}-x_{0}\right\| \\
& \leq M \beta_{0} \eta_{0} g\left(r_{0}\right)=r_{0} g\left(r_{0}\right)<1,
\end{aligned}
$$

therefore, by Banach Lemma, we get

$$
\left\|\Gamma_{1}\right\| \leq \frac{\left\|\Gamma_{0}\right\|}{1-r_{0} g\left(r_{0}\right)}=\left\|\Gamma_{0}\right\| \phi\left(r_{0}\right) .
$$

Moreover,

$$
\left\|\Gamma_{0}\right\|\left\|y_{0}-x_{0}\right\| \omega\left(\left\|y_{0}-x_{0}\right\|\right) \leq \beta_{0} \eta_{0} \omega\left(\eta_{0}\right)=s_{0} .
$$

Using Taylor expansion of $G\left(x_{1}\right)$ about $z_{0}$, we get

$$
\begin{aligned}
G\left(x_{1}\right) & =\int_{0}^{1}\left(G^{\prime \prime}\left(y_{0}+t\left(z_{0}-y_{0}\right)\right)-G^{\prime \prime}\left(y_{0}\right)\right)\left(z_{0}-y_{0}\right)\left(x_{1}-z_{0}\right) d t+G^{\prime \prime}\left(y_{0}\right)\left(z_{0}-y_{0}\right)\left(x_{1}-z_{0}\right) \\
& +\int_{0}^{1} G^{\prime \prime}\left(z_{0}+t\left(x_{1}-z_{0}\right)\right)\left(x_{1}-z_{0}\right)^{2}(1-t) d t+\frac{1}{2} G^{\prime \prime}\left(z_{0}\right)\left(x_{1}-z_{0}\right)^{2} .
\end{aligned}
$$

Therefore,

$$
\begin{aligned}
\left\|\Gamma_{1} G\left(x_{1}\right)\right\| & \leq \phi\left(r_{0}\right)\left\|\Gamma_{0}\right\|\left\|G\left(x_{1}\right)\right\|, \\
& \leq \phi\left(r_{0}\right) \frac{r_{0}^{2}}{2\left(1-r_{0}\right)}\left(1+\frac{r_{0}}{4}\right)\left[\frac{s_{0}}{1+q}\left(\frac{r_{0}^{1+q}}{2^{1+q}}+\frac{1}{2+q}\left(\frac{r_{0}^{2}}{2\left(1-r_{0}\right)}\left(1+\frac{r_{0}}{4}\right)\right)^{1+q}\right)\right. \\
& \left.+\frac{r_{0}}{2}\left(r_{0}+\frac{r_{0}^{2}}{2\left(1-r_{0}\right)}\left(1+\frac{r_{0}}{4}\right)\right)\right] \\
& =\phi\left(r_{0}\right) \psi\left(r_{0}, s_{0}\right) \eta_{0}=\eta_{1} .
\end{aligned}
$$

Using (27), we get

$$
\begin{aligned}
M\left\|\Gamma_{1}\right\|\left\|\Gamma_{1} G\left(x_{1}\right)\right\| & \leq M \phi\left(r_{0}\right)\left\|\Gamma_{0}\right\| \phi\left(r_{0}\right) \psi\left(r_{0}, s_{0}\right) \eta_{0} \\
& \leq r_{0} \phi\left(r_{0}\right)^{2} \psi\left(r_{0}, s_{0}\right)=r_{1}
\end{aligned}
$$

and

$$
\begin{aligned}
\left\|\Gamma_{1}\right\|\left\|\Gamma_{1} G\left(x_{1}\right)\right\| \omega\left(\left\|\Gamma_{1} G\left(x_{1}\right)\right\|\right) & \leq \beta_{0} \eta_{0} \omega\left(\eta_{0}\right) \phi\left(r_{0}\right)^{1+q} \psi\left(r_{0}, s_{0}\right)^{1+q} \\
& \leq s_{0} \phi\left(r_{0}\right)^{2+q} \psi\left(r_{0}, s_{0}\right)^{1+q}=s_{1} .
\end{aligned}
$$


The following recurrence relations are established for $k \geq 1$ using mathematical induction.

(I) $\left\|\Gamma_{k}\right\| \leq \phi\left(r_{k-1}\right)\left\|\Gamma_{k-1}\right\|$,

(II) $\left\|\Gamma_{k} G\left(x_{k}\right)\right\| \leq \phi\left(r_{k-1}\right) \psi\left(r_{k-1}, s_{k-1}\right) \eta_{k-1}$,

(III) $M\left\|\Gamma_{k}\right\|\left\|\Gamma_{k} G\left(x_{k}\right)\right\| \leq r_{k}$,

(IV) $\left\|\Gamma_{k}\right\|\left\|\Gamma_{k} G\left(x_{k}\right)\right\| \omega\left(\left\|\Gamma_{k} G\left(x_{k}\right)\right\|\right) \leq s_{k}$,

(V) $\left\|x_{k}-x_{k-1}\right\| \leq g\left(r_{k-1}\right) \eta_{k-1}$,

Hence, the recurrence relations (I)-(IV) for $k=1$ follow from (25), (27), (28) and (29) respectively. The recurrence relation $(\mathrm{V})$ is proved for $k=1$ in (24). Using mathematical induction, these recurrence relations hold $\forall k \geq 1$.

\subsection{Convergence Theorem}

Theorem 1. Let $r_{0}=M \beta_{0} \eta_{0}<v, s_{0}=\beta_{0} \eta_{0} \omega\left(\eta_{0}\right)$ and assumptions (1)-(4) hold. Then for $\bar{B}\left(x_{0}, R \eta_{0}\right) \subseteq$ $\Omega$, where $R=\frac{g\left(r_{0}\right)}{1-\delta \gamma}$, the sequence $\left\{x_{k}\right\}$ generated by (3) converges to the solution of (1). Moreover, $y_{k}, z_{k}, x_{k+1}, x^{*} \in \bar{B}\left(x_{0}, R \eta_{0}\right)$ and $x^{*}$ is the unique solution in $B\left(x_{0}, \frac{2}{L_{1} \beta_{0}}-R \eta_{0}\right) \cap \Omega$. Then the error bound for iterative scheme verifies:

$$
\left\|x_{k}-x^{*}\right\| \leq g\left(r_{0}\right) \delta^{k} \frac{\gamma^{\frac{(4+q)^{k}-1}{3+q}}}{1-\delta \gamma^{(4+q)^{k}}} \eta_{0} .
$$

Proof. To prove the convergence theorem, we prove that $\left\{x_{k}\right\}$ is a Cauchy sequence. Using (V), we get

$$
\left\|x_{k+1}-x_{k}\right\| \leq g\left(r_{k}\right) \eta_{k} \leq g\left(r_{0}\right) \eta_{k} \leq g\left(r_{0}\right) \prod_{j=0}^{k-1} \phi\left(r_{j}\right) \psi\left(r_{j}, s_{j}\right) \eta_{0} .
$$

Now, we consider

$$
\begin{aligned}
\left\|x_{k+m}-x_{k}\right\| & \leq\left\|x_{k+m}-x_{k+m-1}\right\|+\left\|x_{k+m-1}-x_{k+m-2}\right\|+\ldots+\left\|x_{k+1}-x_{k}\right\| \\
& \leq g\left(r_{0}\right) \prod_{j=0}^{k+m-2} \phi\left(r_{j}\right) \psi\left(r_{j}, s_{j}\right) \eta_{0}+g\left(r_{0}\right) \prod_{j=0}^{k+m-1} \phi\left(r_{j}\right) \psi\left(r_{j}, s_{j}\right) \eta_{0}+\ldots \\
& +g\left(r_{0}\right) \prod_{j=0}^{k-1} \phi\left(r_{j}\right) \psi\left(r_{j}, s_{j}\right) \eta_{0} \\
& \leq g\left(r_{0}\right) \sum_{l=0}^{m-1}\left(\prod_{j=0}^{k+l-1} \phi\left(r_{j}\right) \psi\left(r_{j}, s_{j}\right) \eta_{0}\right)
\end{aligned}
$$

by using Lemma 3 (iii), we get

$$
\begin{aligned}
\left\|x_{k+m}-x_{k}\right\| & \leq g\left(r_{0}\right) \sum_{l=0}^{m-1} \delta^{k+l}\left(\gamma^{\frac{(4+q)^{k+l}-1}{3+q}}\right) \eta_{0} \\
& \leq g\left(r_{0}\right) \delta^{k}\left(\gamma^{\frac{(4+q)^{k}-1}{3+q}}\right) \sum_{l=0}^{m-1}\left(\delta \gamma^{(4+q)^{k}}\right)^{l} \\
& \leq g\left(r_{0}\right) \delta^{k}\left(\gamma^{\frac{(4+q)^{k}-1}{3+q}}\right) \frac{1-\left(\delta \gamma^{(4+q)^{k}}\right)^{m}}{1-\delta \gamma^{(4+q)^{k}}} \eta_{0} .
\end{aligned}
$$


Hence, $\left\{x_{k}\right\}$ is a Cauchy sequence which converges to $x^{*}$ as $k \rightarrow \infty$. Taking $m \rightarrow \infty$ in (32), we get

$$
\left\|x_{k}-x^{*}\right\| \leq g\left(r_{0}\right) \delta^{k} \gamma^{\frac{(4+q)^{k}-1}{3+q}} \frac{1}{1-\delta \gamma^{(4+q)^{k}}} \eta_{0} .
$$

Taking $k=0$ in (33), we get

$$
\left\|x^{*}-x_{0}\right\| \leq \frac{g\left(r_{0}\right)}{1-\delta \gamma} \eta_{0} \leq R \eta_{0}
$$

Hence, $x^{*} \in \bar{B}\left(x_{0}, R \eta_{0}\right)$. Now,

$$
\left\|x_{k+1}-x_{0}\right\| \leq \sum_{i=0}^{k}\left\|x_{i+1}-x_{i}\right\| \leq \sum_{i=0}^{k} g\left(r_{i}\right) \eta_{i} \leq g\left(r_{0}\right) \sum_{i=0}^{k} \eta_{i} \leq R \eta_{0}
$$

and

$$
\left\|y_{k}-x_{0}\right\| \leq\left\|y_{k}-x_{k}\right\|+\left\|x_{k}-x_{0}\right\| \leq \eta_{k}+\sum_{i=0}^{k-1} g\left(r_{i}\right) \eta_{i} \leq g\left(r_{0}\right) \sum_{i=0}^{k} \eta_{i} \leq R \eta_{0} .
$$

Using (22), we get

$$
\left\|z_{k}-x_{0}\right\| \leq\left\|z_{k}-x_{k}\right\|+\left\|x_{k}-x_{0}\right\| \leq\left(1+\frac{r_{0}}{2}\right) \eta_{k}+\sum_{i=0}^{k-1} g\left(r_{i}\right) \eta_{i} \leq g\left(r_{0}\right) \sum_{i=0}^{k} \eta_{i} \leq R \eta_{0} .
$$

Hence, $y_{k}, z_{k}, x_{k+1} \in \bar{B}\left(x_{0}, R \eta_{0}\right)$.

To show the uniqueness of $x^{*}$, let $z^{*} \in B\left(x_{0}, \frac{2}{M \beta}-R \eta_{0}\right) \cap \Omega$ be such that $G\left(z^{*}\right)=0, z^{*} \neq x^{*}$. Then $0=G\left(z^{*}\right)-G\left(x^{*}\right)=\int_{0}^{1} G^{\prime}\left(x^{*}+t\left(z^{*}-x^{*}\right)\right) d t\left(z^{*}-x^{*}\right)=P\left(z^{*}-x^{*}\right)$, where, $P=\int_{0}^{1} G^{\prime}\left(x^{*}+\right.$ $\left.t\left(z^{*}-x^{*}\right)\right) d t$. Now,

$$
\begin{aligned}
\left\|I-\Gamma_{0} P\right\| & \leq\left\|\Gamma_{0}\right\| \int_{0}^{1}\left\|\left(G^{\prime}\left(x^{*}+t\left(z^{*}-x^{*}\right)\right)-G^{\prime}\left(x_{0}\right)\right)\right\| d t \\
& \leq \frac{M \beta}{2}\left(\left\|x^{*}-x_{0}\right\|+\left\|z^{*}-x_{0}\right\|\right) \\
& <\frac{M \beta}{2}\left(R \eta_{0}+\frac{2}{M \beta}-R \eta_{0}\right) \\
& =1
\end{aligned}
$$

Therefore, $\left\|I-\Gamma_{0} P\right\|<1$. Thus, $P^{-1}$ exists by Banach Lemma and hence $z^{*}=x^{*}$.

\section{Numerical Experience}

In this section, different numerical examples are solved in order to corroborate the theoretical results obtained and the efficiency of our approach.

Example 1. Consider nonlinear integral equation

$$
G(x)(s)=x(s)-1+\int_{0}^{1} H(s, t)\left(\frac{3}{5} x(t)^{7 / 3}+\frac{6}{15} x(t)^{3}\right) d t,
$$

where $s \in[0,1], x \in \Omega=B(0,2) \subset X$. 
Clearly,

$$
\left\|G^{\prime \prime}(x)-G^{\prime \prime}(y)\right\| \leq \frac{7}{30}\|x-y\|^{1 / 3}+\frac{3}{10}\|x-y\|
$$

where $\omega(\mu)=\frac{7}{30} \mu^{1 / 3}+\frac{3}{10} \mu$ and $q=\frac{1}{3}$. Therefore, neither the Lipschitz nor the Hölder condition hold but the $\omega$-condition holds. Taking $x_{0}(t)=1$, all the assumptions are satisfied. Therefore, the existence and uniqueness balls for integral equation are given by $\bar{B}\left(x_{0}, 0.21621\right)$ and $B\left(x_{0}, 1.2939\right)$, respectively. The values of the sequences $\left\{r_{k}\right\},\left\{s_{k}\right\}$ and $\left\{\eta_{k}\right\}$ are given in Table 1 .

Table 1. The values of $r_{k}, s_{k}$ and $\eta_{k}$.

\begin{tabular}{cccc}
\hline $\mathbf{k}$ & $\boldsymbol{r}_{\boldsymbol{k}}$ & $\boldsymbol{s}_{\boldsymbol{k}}$ & $\eta_{\boldsymbol{k}}$ \\
\hline 0 & 0.24527 & $5.1729 \times 10^{-2}$ & 0.18519 \\
1 & $7.7009 \times 10^{-4}$ & $2.126 \times 10^{-5}$ & $4.1532 \times 10^{-4}$ \\
2 & $6.8009 \times 10^{-17}$ & $8.359 \times 10^{-23}$ & $3.665 \times 10^{-17}$ \\
3 & $3.6373 \times 10^{-82}$ & $7.8181 \times 10^{-110}$ & $1.9601 \times 10^{-82}$ \\
4 & $1.5916 \times 10^{-408}$ & $5.5956 \times 10^{-545}$ & $8.5771 \times 10^{-409}$ \\
5 & $2.5535 \times 10^{-2040}$ & $1.0509 \times 10^{-2720}$ & $1.3761 \times 10^{-2040}$ \\
\hline
\end{tabular}

The error bounds for $x^{*}$ are presented in Table 2.

Table 2. Error bounds.

\begin{tabular}{cc}
\hline $\mathbf{k}$ & $\left\|x_{k}-x^{*}\right\|$ \\
\hline 0 & $4.8381 \times 10^{-4}$ \\
1 & $4.9181 \times 10^{-15}$ \\
2 & $3.4921 \times 10^{-62}$ \\
3 & $5.2372 \times 10^{-266}$ \\
4 & $9.3095 \times 10^{-1149}$ \\
5 & $1.6043 \times 10^{-4973}$ \\
\hline
\end{tabular}

Example 2. Consider nonlinear integral equation

$$
G(x)(s)=x(s)-f(s)-\lambda \int_{0}^{1} \frac{s}{s+t} x(t)^{2+q} d t
$$

where, $x, f \in C[0,1], \lambda \in \mathbb{R}$ and $s \in[0,1]$.

Clearly,

$$
\left\|G^{\prime \prime}(x)-G^{\prime \prime}(y)\right\| \leq|\lambda| \log 2(1+q)(2+q)\|x-y\|^{q} .
$$

Here $\omega(\eta)=|\lambda| \log 2(1+q)(2+q) \eta^{q}$ Clearly, Lipschitz condition fails for $q \in(0,1)$ but Hölder condition holds. Taking $x_{0}=x_{0}(s)=1, q=\frac{1}{5}, \lambda=\frac{1}{4}$, and $f(s)=1$, all the assumptions are satisfied. Therefore the existence and uniqueness balls for integral equation is given by $\bar{B}\left(x_{0}, 0.3174\right)$ and $B\left(x_{0}, 2.3879\right)$ respectively. The values of $\left\{r_{k}\right\},\left\{s_{k}\right\}$ and $\left\{\eta_{k}\right\}$ are given in Table 3 . 
Table 3. The values of $r_{k}, s_{k}$ and $\eta_{k}$.

\begin{tabular}{cccc}
\hline $\mathbf{k}$ & $c_{\boldsymbol{k}}$ & $\boldsymbol{d}_{\boldsymbol{k}}$ & $\eta_{\boldsymbol{k}}$ \\
\hline 0 & 0.20704 & 0.16052 & 0.28005 \\
1 & $3.4155 \times 10^{-4}$ & $6.9698 \times 10^{-5}$ & $3.5373 \times 10^{-4}$ \\
2 & $1.1985 \times 10^{-18}$ & $3.1433 \times 10^{-22}$ & $1.2408 \times 10^{-18}$ \\
3 & $6.1808 \times 10^{-91}$ & $5.6532 \times 10^{-109}$ & $6.399 \times 10^{-91}$ \\
4 & $2.255 \times 10^{-452}$ & $1.0637 \times 10^{-542}$ & $2.3347 \times 10^{-452}$ \\
5 & $1.4579 \times 10^{-2259}$ & $2.509 \times 10^{-2711}$ & $1.5094 \times 10^{-2259}$ \\
\hline
\end{tabular}

The error bounds for $x^{*}$ are presented in Table 4 .

Table 4. Error bounds.

\begin{tabular}{cc}
\hline $\mathbf{k}$ & $\left\|x_{k}-x^{*}\right\|$ \\
\hline 0 & $4.004 \times 10^{-4}$ \\
1 & $6.3045 \times 10^{-16}$ \\
2 & $3.9701 \times 10^{-65}$ \\
3 & $2.1201 \times 10^{-271}$ \\
4 & $2.2551 \times 10^{-1137}$ \\
5 & $4.3337 \times 10^{-4774}$ \\
\hline
\end{tabular}

\section{Conclusions}

In this study, we present the semilocal convergence for an iterative scheme that reach order of convergence five. We obtained the theoretical results by constructing the recurrence relations that describe this algorithm that is proven to have a very efficient behavior. The hypotheses we set are under weaker conditions than the used in previous studies and allow us to obtain competitive error bounds. Finally, applied problems are solved involving nonlinear integral equations and big size nonlinear systems. The convergence balls defining the existence domain were obtained for the considered examples.

Author Contributions: Conceptualization and methodology, S.S. and E.M.; software and validation, D.K.G. and A.K.; Formal analysis E.M.; investigation, D.K.G; writing—original draft preparation, S.S.; writing-review and editing, S.S. and A.K.; supervision, E.M. and D.K.G. All authors have read and agreed to the published version of the manuscript.

Funding: This research was partially supported by Ministerio de Economía y Competitividad under grant PGC2018-095896-B-C22.

Conflicts of Interest: The authors declare no conflict of interest.

\section{References}

1. Traub, J.F. Iterative Methods for the Solution of Equations; Prentice-Hall: Englewood Cliffs, NJ, USA, 1964.

2. Argyros, I.K.; Hilout, S.; Tabatabai, M.A. Mathematical Modelling with Applications in Biosciences and Engineering; Nova Publishers: New York, NY, USA, 2011.

3. Argyros, I.K.; Hilout, S. Numerical Methods in Nonlinear Analysis; World Scientific Publ. Comp.: Singapore, 2013.

4. Hernández, M.A. Chebyshev's approximation algorithms and applications. Comput. Math. Appl. 2011, 41, 433-445. [CrossRef]

5. Amat, S.; Hernández, M.A.; Romero, N. A modified Chebyshev's iterative method with at least sixth order of convergence. Appl. Math. Comput. 2008, 206, 164-174. [CrossRef]

6. Argyros, I.K.; Ezquerro, J.A.; Gutiérrez, J.M.; Hernández, M.A.; Hilout, S. On the semilocal convergence of efficient Chebyshev-Secant-type methods. J. Comput. Appl. Math. 2011, 235, 3195-3206. [CrossRef]

7. Hueso, J.L.; Martínez, E. Semilocal convergence of a family of iterative methods in Banach spaces. Numer. Algorithms 2014, 67, 365-384. [CrossRef] 
8. Zhao, Y.; Wu, Q. Newton-Kantorovich theorem for a family of modified Halley's method under Hölder continuity conditions in Banach space. Appl. Math. Comput. 2008, 202, 243-251. [CrossRef]

9. Parida, P.K.; Gupta, D.K. Recurrence relations for a Newton-like method in Banach spaces. J. Comput. Appl. Math. 2007, 206, 873-887. [CrossRef]

10. Parida, P.K.; Gupta, D.K. Recurrence relations for semilocal convergence of a Newton-like method in Banach spaces. J. Math. Anal. Appl. 2008, 345, 350-361. [CrossRef]

11. Cordero, A.; Ezquerro, J.A.; Hernández, M.A.; Torregrosa, J.R. On the local convergence of a fifth-order iterative method in Banach spaces. Appl. Math. Comput. 2015, 251, 396-403. [CrossRef]

12. Argyros, I.K.; Khattri, S.K. Local convergence for a family of third order methods in Banach spaces. J. Math. 2014, 46, 53-62.

13. Argyros, I.K.; Hilout, A.S. On the local convergence of fast two-step Newton-like methods for solving nonlinear equations. J. Comput. Appl. Math. 2013, 245, 1-9. [CrossRef]

14. Argyros, I.K.; George, S.; Magreñán, A.A. Local convergence for multi-point-parametric Chebyshev-Halleytype methods of higher convergence order. J. Comput. Appl. Math. 2015, 282, 215-224. [CrossRef]

15. Wang, X.; Kou, J.; Gu, C. Semilocal convergence of a class of Modified Super-Halley Methods in Banach Spaces. J. Optim. Theory Appl. 2012, 153, 779-793. [CrossRef]

16. Argyros, I.K.; Magreñán, A.A. A study on the local convergence and the dynamics of Chebyshev-Halley-type methods free from second derivative. Numer. Algorithms 2016, 71, 1-23. [CrossRef]

17. Wu, Q.; Zhao, Y. Newton-Kantorovich type convergence theorem for a family of new deformed Chebyshev method. Appl. Math. Comput. 2008, 192, 405-412. [CrossRef]

18. Martínez, E.; Singh, S.; Hueso, J.L.; Gupta, D.K. Enlarging the convergence domain in local convergence studies for iterative methods in Banach spaces. Appl. Math. Comput. 2016, 281, 252-265. [CrossRef]

19. Kumar, A.; Gupta, D.K.; Martínez, E.; Singh, S. Semilocal convergence of a Steffensen-type method under weak Lipschitz conditions in Banach spaces. J. Comput. Appl. Math. 2018, 330, 732-741. [CrossRef]

20. Singh, S.; Gupta, D.K.; Martínez, E.; Hueso, J.L. Semilocal convergence analysis of an iteration of order five using recurrence relations in Banach spaces. Mediterr. J. Math. 2016, 13, 4219-4235. [CrossRef]

21. Khoshgam, Z.; Ashrafi, A. A new modified structured BFGS algorithm by utilizing a new modified secant equation. J. Nonlinear Funct. Anal. 2019, 2019, 30.

22. Akram, M.; Chen, J.W.; Dilshad, M. Generalized Yosida approximation operator with an application to a system of Yosida inclusions. J. Nonlinear Funct. Anal. 2018, 2018, 17.

23. Domoshnitsky, A. Sturm theorems and distance between adjacent zeros for second order integro-differential equations. J. Nonlinear Var. Anal. 2018, 2, 155164. 